\title{
Case Report: Lymphatic Drainage Resolves Toe Walking Gait in a Boy with Autism Spectrum Disorder
}

\author{
Susan Vaughan Kratz, OTR, CST-D* \\ Registered Occupational Therapist, Diplomate CranioSacral Therapy, Special Therapies, Inc., USA
}

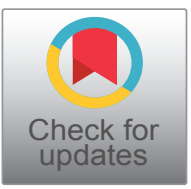

*Corresponding author: Susan Vaughan Kratz, OTR, CST-D, Registered Occupational Therapist, Diplomate CranioSacral Therapy, Special Therapies, Inc.,1720 Dolphin Drive, Unit B, Waukesha, Wisconsin 53186, USA, Tel: 262-347-2222

\begin{abstract}
Objective: Describe how lymphatic drainage (LD) resolved obligatory toe walking gait and stance in a boy with Autism Spectrum Disorder (ASD). The treatment plan was generalized from an identical treatment given to a collegiate athlete with chronic spasms of gastronemii.

Design: Review of medical record; photography evidence.

Intervention: A single treatment of LD. No literature suggests a fluid anatomy model for manual therapies as treatment options for toe walking. This case highlights an effective method discovered to quickly reverse functional deformities despite several years of traditional pediatric interventions for toe walking in ASD.

Results: Full weight bearing contact of bilateral plantar surfaces was gained in stance immediately. Active-assisted range of motion and progressive resistance training began to progress only after this breakthrough. Gains have since been made in gait and stance normalization, and foot kinetics. More invasive interventions (serial casting; reconstructive surgery) have been avoided. Gains sustained as evidenced in one year follow up.

Conclusion: Emerging paradigm of lymphatic and interstitial anatomy provides novel treatment to change the functional status of muscle tissue and tone. Changes in passive and active ankle range of motion were a direct result of mobilizing and evacuating fluids of the gastrocnemii via lymphatic pathways. Spontaneous muscle softening and Achilles tendon lengthening happened instantly. An extremely light touch and pressure input from the therapist is required to perform lymphatic drainage. Lymphatic drainage massage holds promise for further use and study with issues such as toe walking gait and other orthopedic populations.
\end{abstract}

\section{Keywords}

Toe walking, Gastrocnemius spasms, Lymphatic drainage massage, Autism spectrum disorders

\section{Introduction}

Although toe-walking is considered to be within the normal gait spectrum of childhood, it becomes abnormal when persisting past two or three years of age $[1,2]$. The pattern of toe walking as a bilateral gait abnormality where strides are taken with a toe-to-toe pattern, normal heel strike is absent and full weight bearing occurs only at the forefoot. Concurrent issues of pronation with subtalar strain or collapse, and an imbalance of rear-foot to forefoot dynamics are also common. Active dorsiflexion has varying degrees of weakness and range of motion $[2,3]$. The prevalence of toe walking at age 5.5 years is $2 \%$ in typically developing children, and $41 \%$ in children with a neuropsychiatric diagnosis or developmental delays $[4,5]$.

Idiopathic toe walking is a term given when these structural and movement disturbances are present without a discernable etiology $[1,6]$. On the other hand, known etiologies may be cerebral palsy or other neurological insult, a congenital contractures, or paralytic muscular disorders such as Duchenne Muscular Dystrophy [7-9]. Differential diagnosis is best reached through careful history and clinical exam of both neurological and orthopedic factors $[4,10]$. Toe-walking gait is a common behavioral feature extensively reported in children with Autism Spectrum Disorder (ASD), which often occurs with other neurological symptoms, including ataxia, hypotonia, variable stride length and duration, incoordination, postural abnormalities in the head and trunk, reduced plantarflexion and increased dorsiflexion [11-17].

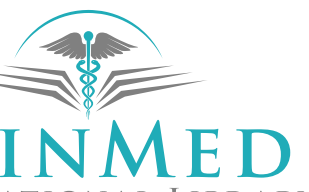

INTERNATIONAL LIBRARY 
Toe-walking has also been theorized to be a compensatory gait pattern of advantage for patients with upper motor neuron injury and distal lower extremity weakness by requiring less ankle plantar flexion and dorsiflexion, and knee extensor strength than normal heel-toe walking [18]. Sensory disturbances with maladaptive use of proprioception, tactile, and vibration input could be a complicating factor in normal heel-strike walking $[19,20]$. Walking surface has also been shown to possibly alter gait patterns in both children with toe-walking gait and typically developing children [21].

Hypotonia and retained primitive reflexes have also been cited as strong correlates to postural development [22]. An association between toe-walking gait and the presence of components of the tonic labyrinthine reflex has been recognized, suggesting that toe walking was more a function of motor control [11]. Children with ASD had significantly greater joint mobility, more gait abnormalities and variations, and walked later than their non-autistic peers [23-25]. One study investigated the incidence of persistent toe walking and tight Achilles tendons in children with ASD to be $20 \%$ and $12 \%$ respectively [26]. Several studies refer to cerebellar and basal ganglia involvement as possible etiologies for motor differences in ASD [15,17,23,27]. Long term consequences and complications of prolonged toe-walking gait include secondary contractures and musculoskeletal deformity, and the cost of prolonged therapies as well as surgery $[26,28]$.

\section{Available Treatments Options for Toe-Walking Gait}

Current best practice in treating toe-walking gait, regardless of etiology, include: Observation, conservative and traditional physical medicine methods of stretching and progressive resistive exercises, sensory-motor strategies, serial casting, orthotics, chemical denervation, and surgical lengthening of the gastroc-soleus-Achilles complex [4,13,29-31]. Intervention options are typically driven by clinical reasoning based on age, underlying etiology, cognitive ability, and the severity of tendon contracture.

\section{Serial casting}

Serial casting has been long advocated for toe-walking despite a lack of large scale evidence across diagnostic groups or strong evidence of long term outcomes $[28,32,33]$. The goal of serial casting is to achieve sustained stretch of the [muscle] tissue of the gastroc/soleus complex and the Achilles tendon in order gain passive/active dorsiflexion. Positive effects from serial casting have been demonstrated which makes this method a common treatment of choice for persistent toe walking not responding to other interventions [34,35].

\section{Orthoses}

The use of ankle-foot orthosis may not control toe walking as well as casting but is less restrictive and more accepted by children and their parents, with similar out-of-brace effects [29]. Ankle-foot-orthoses can restrict toe walking when worn, but children may revert to equinus gait once the orthosis is removed [4]. One recent case report describes how a combination of serial casting to gain passive dorsiflexion followed with the use of ankle-foot orthosis assisted the improvement of heel-toe gait for a child with autism [35].

\section{Surgery}

Persistent toe-walking gait is a common reason for a referral to a pediatric orthopedic surgeon [1]. Post-surgical management involves participation in physical medicine activities to maximize surgical outcomes, but language and sensory motor challenges could have an impact on outcome of post-surgical rehabilitation. Clinical outcome studies have been on either smaller samples or with varied effects, but as with serial casting, benefits of surgical lengthening of Achilles tendon have been documented [36-38]. "Surgical treatment may influence the outcome, but indications for surgery need to be clarified" [9]. "Surgical treatment of toe walking should be reserved for the few cases with a fixed ankle-joint contracture" [39].

\section{Behavioral and sensory entrainments}

Behavioral shaping through specific teaching and/ or sensory-based entrainment methods have been illustrated and demonstrated positive effects in small sample groups of children with ASD [40,41]. Kinesiology taping (elastic-fibered athletic tape to stabilize joint articulations and correct alignment) has been suggested to be used to alter toe-walking gait $[42,43]$.

The wearing of high top [tennis] shoes or hiking boots as daily wearing apparel has a long history of recommended use in pediatric therapy settings as a deterrent for habitual toe walking. Unfortunately, though it appears that passive dorsiflexion might be approximated, without the use of radiology, true ankle and foot alignment and position cannot be assured. Foot wear that are heavier in weight have also been recommended as proprioceptive enhancement to promote plantar surface contact with floor. There is no empirical data to support such use or guide clinical practice in foot apparel recommendations. Similar to the problem seen with orthosis use, the reverting back to toe walking gait when shoes are not worn is an issue also not yet addressed.

\section{Manual therapies}

Manual and stretch therapies are akin to the goals of serial casting, (to increase and maintain the length of Achilles tendon, gastrocnemius and/or soleus mus- 
cles to pain passive dorsiflexion range of motion). Manual therapies and massage methods have been shown to relieve spasms and are theorized to detox muscles for the athlete sports performance, though there is limited high-ranking evidence to promote such methods in injuries $[44,45]$. No literature suggests that toe walking gait might be a result of chronic muscle spasms.

\section{The Hypothesis}

Although adequate information of existing traditional interventions for toe-walking gait for functional neurological disorders is available in scientific reviews, there is no mention of the use of manual therapies specific to a "fluid model of human anatomy" $[10,46,47]$. There can be more than mere muscles and tendons that are tight and restricted created by a chronic posture. Fascia fields, blood vessels, interstitium, skin, and even soft tissue adhesions to bone can all potentially limit the lengthening of the gastroc/soleus to allow for neutral ankle position in stance and gait.

Lymphatic drainage pathways assist the movement and exchange of interstitial as well as lymphatic fluids. The inability to exchange fluids to purify muscle may have caused chronic structural restrictions and fibrotic stiffness as a result of cellular terrain inflammation. Coulda build-up of lactic acid (or other toxins) within muscle and soft tissues preventing normal drainage of lymphatic and interstitial fluids, and thus prevent muscle relaxation and interfere with neutral dorsiflexion?

\section{Method}

The use of Chikly method of lymphatic drainage massage was applied generalizing the success of treating a college athlete for chronic leg spasms using the same intervention $[48,49]$. This contemporary approach to facilitating the evacuation of stagnant cellular terrain follows specific anatomical pathways, lymphatic mapping, and accuracy in sending lymph fluids to corresponding nodes responsible for drainage of specific body regions.

A major distinction of this method is the extreme lightness and non-aggressive touch and strokes to the lymph tissues the therapist employs and following a specific direction of lymphatic anatomy. Tissue-directed treatment dictates that the therapist waits for signals of fluid movement and moves in sync with the rhythm of the body's lymph physiology. It is an art form to learn though training is available for any profession. A milking of lymph fluids through the congested channels of the lymph node pathways cephalad describes the technique. Deep tissue massage or mobilization was not used in this case.

Competency of application of this method is recommended through professional training workshops $[48,49]$.

\section{Case Report}

\section{Patient information}

This case is of a young boy who was six and a half years old at the time the treatment was applied. He held a formal diagnosis of Autism Spectrum Disorder since the age of 30 months and was previously involved with an in-home psychology-based behavioral training program. His parents sought additional services with occupational therapy to maximize his development and global pervasive dysfunction. (He was also receiving speech and occupational therapy services at his local school). Medical background was positive for possible birth trauma during labor and delivery at a gestation age of 37 weeks. The family also sought alternative options to explore biological etiologies and treatments for the ASD and underlying neurological symptoms.

\section{Clinical findings}

Severe dyspraxia, hypotonia, sensory processing dysfunction with mal-adaptation to proprioception, as well as severe language processing dysfunction were key features of his neuro-behavioral presentation. Assessment conclusions were reached through subjective appraisal, response to treatment interventions, and clinical observations since formal testing was not possible due to lack of functional expressive and receptive language skills. Toe-walking gait and persistent plantar-flexed stance were but one aspect of the therapy planning needs identified by the author (an occupational therapist with 36 years of clinical experience with autism and other neurological disorders).

Noting his initial evaluation in the medical record at the age of four-and-a-half years, several primitive reflexes were retained: Tonic labyrinthine reflex, hyper-responsivity of tendon reflexes in legs, strong extensor thrust with deep tendon stretch/tap, hyper-reactive plantar reflex. Reflexes seemed only to strengthen with repeated direct stimulation to feet. This made traditional manual [stretching] ineffective and contributed to reinforcing the abnormal plantar-flexed ankle pattern. Toe walking and a fixed plantar-flexion stance had been life-long featuresfrom the time he was ambulatory and was becoming increasingly problematic upon skeletal integrity as he aged. (Figure 1: Before Treatment).

Range of motion of ankles was relatively equal between right and left sides. He generally stood, walked, and even jumped in full plantar flexion $\left(50^{\circ}\right)$. He lacked active dorsiflexion thus standing dynamic activities was problematic. No active inversion or eversion was noted. He maintained stereotypic postures ofeither sitting crossed-legged on floor or standing on toes. The primary concern in the original and annual treatment plans was gaining neutral dorsal flexion in order for him to participate in more functional dynamic standing and balance activities. 


\section{BEFORE TREATMENT:}

Photo 1 and 2 image captured typical rigid and static planter-flexion stance when patient was standing in reception area waiting to enter clinic. Candid photo taken without usual cues or instructions to 'put feet down. Photos capture the persistent plantar reflex with inability to achieve neutral dorsiflexion in weight bearing stance.

Photo 3: Photo capture of toe-walking gait pattern, representing of the life-long persistent, reflexive, rigid, and obligatory plantar flexion.
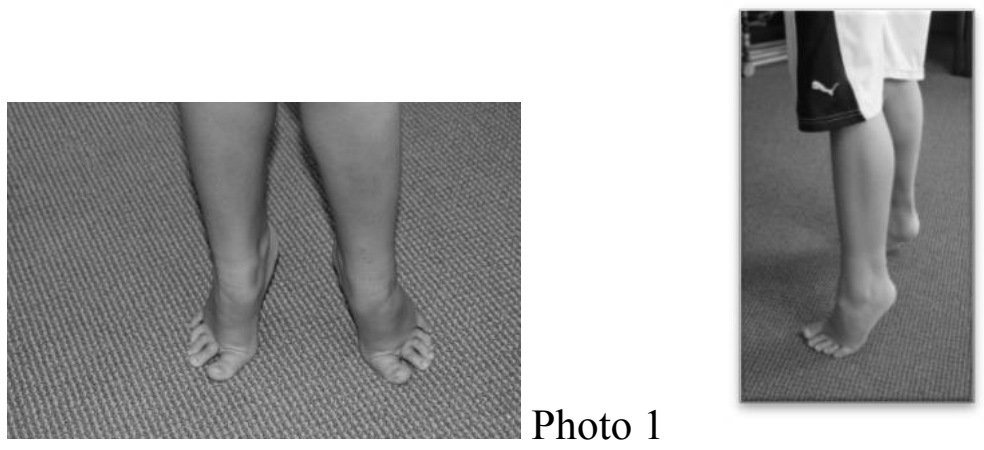

Photo 2

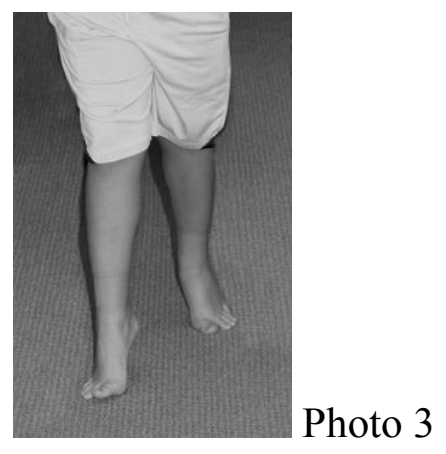

Figure 1: Baseline photos of typical stance and gait on day of treatment. Patient is age $61 / 2$ years.

Mobility skills of basic ambulation emerged on time, but he balance and motor planning difficulties prevented him from gaining skills of crouching, bending to touch toes, jumping or bouncing, or push heavy items utilizing full body recruitment (to encourage flat foot contact on floor). Score on the Pediatric Evaluation of Disability Inventory (PEDI) in Mobility subsection:

Raw Score 52 of 59 possible

\section{$\leq 10 \%$ for age Normative Standard Score}

\section{Timeline and therapeutic interventions trialed}

Addressing the toe walking was not a primary treatment objective when he began participating in outpatient therapy at the age of 4.5 years. After turning 5 years of age, toe walking patterns had shown no indications of change and the following treatments were then incorporated into the treatment plan:

- Therapeutic Exercises and Activities: Sensory motor framework and scaffolded to create opportunities for active weight-bearing pressure into plantar surfaces of feet, push off fully from foot surface, normalize weight bearing in standing, normalize weight shifting, kinesiotaping, night-wear splinting.

- Manual Therapies - Muscle and tendon stretching; joint mobilization; acupressure; myofascial release; craniosacral therapy.

- Activities of Daily Living: Use of high top tennis shoes.

- Cognitive/behavior entrainment: Imposed downward pressure through body to promote dorsiflexion, singing directives for foot placement, reward system, im- proving systemic hypotonia, improving developmental dyspraxia.

At the age of 5.5 years, his first annual therapy review noted:

- Significant toe walking persisted and had become a long term orthopedic concern; ambulation on rough terrain remained compromised; ascending/ descending full flight of stairs was more adversely affected, and a complete collapse of plantar arches noted.

- No change in passive or active dorsiflexion.

- Higher levels of balance had not progressed due to toe walking.

- Still can't walk and carry an object of any weight; couldn't stand for longer than one minute without restlessness.

Over a span of two and a half years, passive and active range of motion at ankles were tracked as unchanging of -30 passive. The annual progress report at age 6.5 years documented theongoing use of high top (tennis) shoes which appeared to prevent extreme toe walking pattern of plantar flexion. The shoes reduced plantar flexion severity in stance and gait, however, full contact in neutral dorsiflexion was not confirmed within the confines of these shoes. Subtalar neutral control and stability were not achieved. In barefoot stance and gait, the toe walking not only resumed, it intensified bilaterally with more pronounced pronated subtalar joints. Collapse of tarsal arches wasalso noted to be more pronounced due to increased height and weight from his growth. 
Verbal directives and behavioral shaping were ineffective at changing toe walking gait for more than two to three steps. Attempts to gain passive ankle range of motion through stretching and muscle mobilization was becoming more futile as extensor thrust patterns were increasing with manual contact with his feet. Serial casting and surgery were now being considered due to ankle deformities becoming more fixed, even though his cognitive and language deficits were problematic for best prognosis of participating in the therapeutic activities associated with these interventions.

Through various work from alternative medicine approaches, it was opinioned that toxic levels of copper, lead, aluminum and glyphosate both in the circulatory system and deeply within body tissue. Various natural detoxification efforts were trialed over the span of several years. No effect upon his gait or stance occurred with these treatments. Toe walking persisted (when not in high top tennis shoes) for a full year beyond the active portions of most of the biomedical and detoxification endeavors. He remained on various diet and supplement programming. No genetic or muscle biopsy testing had been conducted to determine etiologic aspect of hypertrophied and fibrous (gastrocnemius) muscles.

\section{Description of treatment strategy and outcomes}

Shortly after this patient's annual therapy progress report (at age 6.5 years) manual lymphatic drainage was applied to both lower extremities. A modified Chikly sequence was administered due to the unpredictability of the patient's cooperation and attention span for conducting the full protocol. The progression of treatment was identical in both legs. The inguinal nodes were first evacuated, followed by upper leg

\section{Photo reproduction of sequence of lymphatic drainage used.**}

A major differentiation between this method and typical soft tissue massage is the lightness of the touch and following a specific direction of lymphatic anatomy. Chikly's method of fluid evacuation from congested and firm lymph nodes, the strokes are quite gentle and non-aggressive. Tissue-directed treatment dictates the therapist waits for signals of fluid movement and moves in sync with the rhythm of body's lymph physiology. A milking of lymph fluids through the congested channels of lymph pathways cephalad describes the technique.

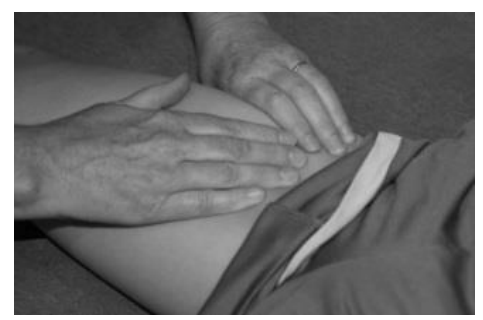

Step One: Evacuating Inguinal nodes, followed by superficial vessels and nodes of femur

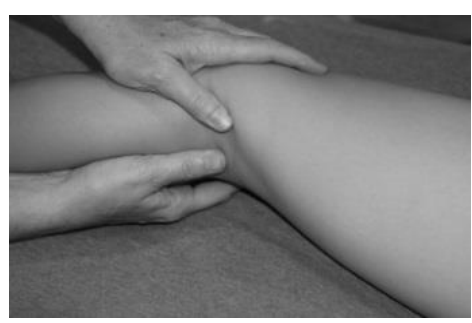

Step Two: Evacuating the engorged popliteal nodes located imbedded in fat of popliteal fossa, followed by lymphatic drainage of superficial vessels of the lower leg.

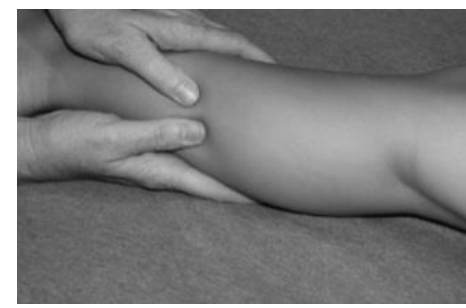

Step Three: Evacuating deep lymph chains: anterior and posterior tibia region

Figure 2: Treatment Sequence. ${ }^{* *}$ This is a descriptive summary only. This sequence should not be construed as the exact Chikly technique. Training for the individual therapist is highly recommended. 
lymphatic pathways evacuated of lymph fluids. Moving distally to popliteal lymph nodes, it became apparent that both sides had fully engorged nodes with no appreciable movement of lymph fluids. Gentle and repetitive manual 'milking' of these fibrous, engorged nodes ensued (in a cephalad direction to follow the normal lymph direction of flow). After approximately 50-60 repetitions, these engorged nodes 'emptied' and became soft, pliable and more dynamic to reception of fluids. Both superficial and deep lymph nodes within gastroc/soleus muscle tissue and surrounding tibia/fibia were treated within corresponding lymphatic pathways (located near adjacent blood vessels) (Figure 2: Treatment Sequence).

\section{Clinical Outcome}

An immediate positive effect of full relaxation and softening of bilateral gastrocnemius/soleus complex was achieved, evidenced through palpation. Flexibility and suppleness of the muscles returned as all fluid flow

\section{Treatment Outcome - following a single treatment session of Manual Lymphatic Drainage}
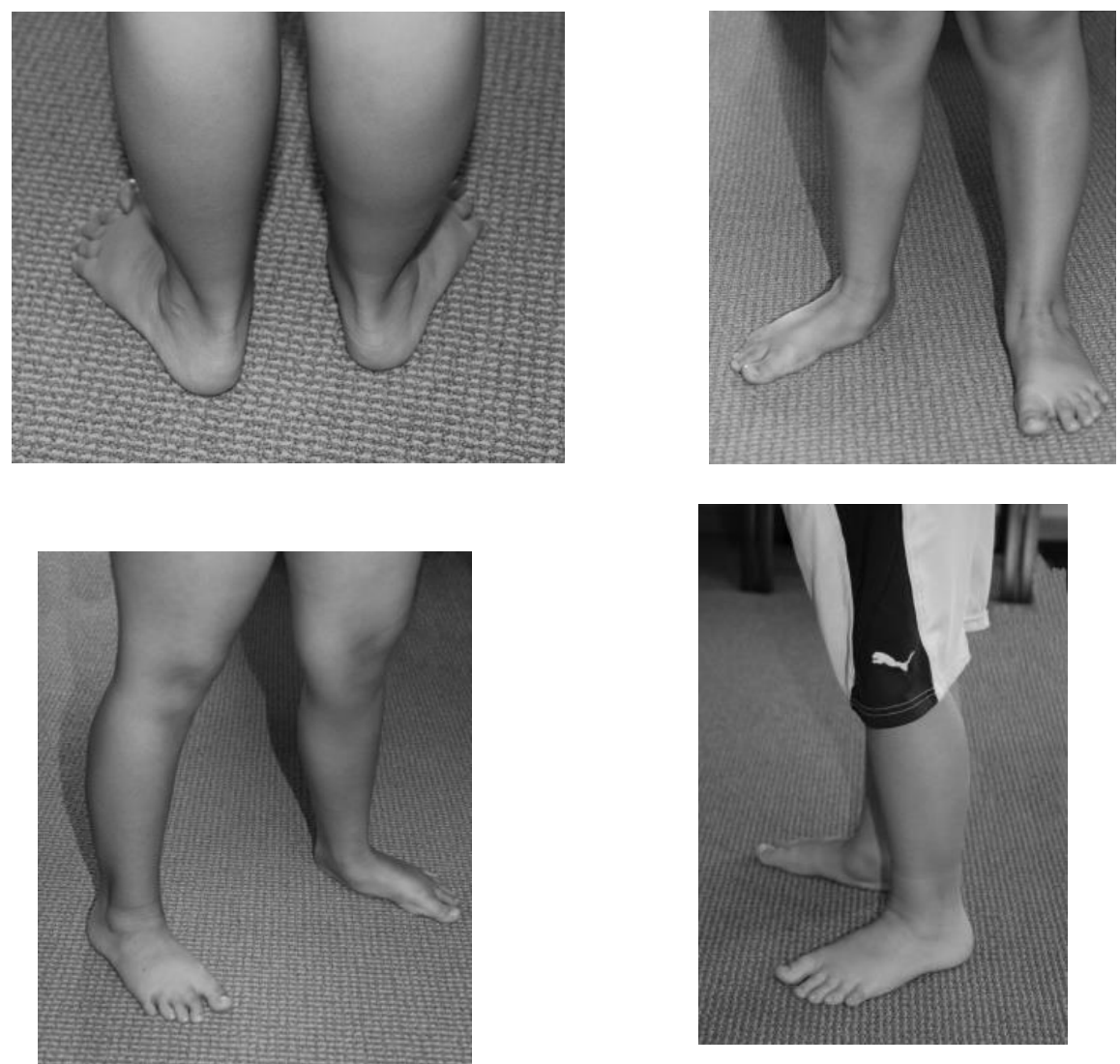

Spontaneous weight-bearing stance without verbal or physical prompts.

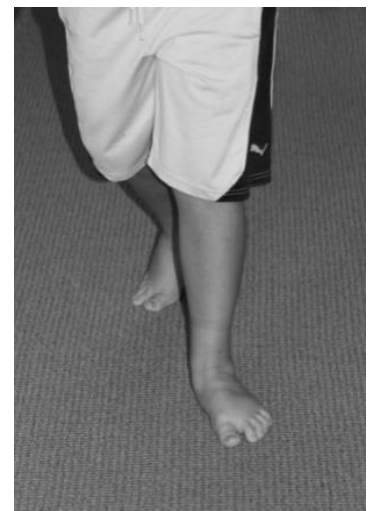

Post treatment of gait pattern. Neutral dorsiflexion achieved in spontaneous weight bearing and foot placement on floor. [Best available photo of gait as cooperation was waning].

Figure 3: Outcome of one session of lymphatic drainage massage: Reflect spontaneously-achieved neutral dorsiflexion in both standing and gait immediately following treatment. 
(blood, lymph, and interstitium) improved in quality. The treatment process took 10-15 minutes to apply to each leg. Immediately upon descending the treatment table, the patient spontaneously stood with full-foot placement on the floor with bilateral neutral dorsiflexion without duress. This was the first time either foot was clinically observed to make complete plantar surface contact. Outcomes were captured in a photograph (Figure 3: After Treatment).

\section{Traditional therapies became more effective}

Beyond the outcomes of this new discovery, in the year since this event, as he continued with long term intensive occupational therapy, more traditional treatments have proven effective. He can now perform active-assisted range of motion exercises (for dorsi/plantar flexion and pronation/supination) and progressive resistance training for up to 50-75 repetitions. Prior to this range of motion achievement of the ankles, he showed no interest or investment of therapeutic activities that involved goals of changing toe walking. However, since this time he shows active investment and understanding in carrying out active-assisted range of motion and therapeutic exercises. This achievement has also contributed to the boy's improved active participation and attention span in postural and core strengthening in both standing and walking.

Sensory motor bombardment in gross motor tasks such as productive marching, stomping, and dynamic weight shifting through all planes are now yielding measurable improvement in his adaptive reactions. He can now participate in ankle strengthening exercises in his home program in the year following this discovering. Ankles still lack complete subtalar stability (supported now with sole inserts in shoes) and dynamic foot kinetics between forefoot and hind-foot. He continues to have pronated stance (without the assist of orthotics). Assisted lateral weight shifts with guidance are now achieved and dynamic tarsal arches can now be attained with assistance.

Previous clinical trials of traditional myofascial mobilization, muscle stretching, reflex inhibition, forced weight bearing, ankle positioning in shoes and other interventions yielded no or few lasting results. Obligatory toe walking had previously contributed to the onset of tarsal and subtalar malalignment contractures, but with the progress of gaining relaxation and softening of the gastronemii, these contractures have been rapidly reversed. Now ankle strengthening and gaining heel strike remain to be gained and the prognosis of normalized gait is good.

Retest of Pediatric Evaluation of Disability Inventory (PEDI) in the subsection, Age $71 / 2$ years:

\section{Mobility Skills}

Raw Score: 59 of 59 possible

\section{$55 \%$ for age Normative Standard Score}

Serial casting and surgery are no longer considered needed at this time. Ongoing monitoring of ankle range of motion, tendon contractions, foot/ankle kinetics, and advancements in higher postural and balance reactions remain for of the long term treatment plan. Status at one year post lymphatic treatment is highlighted (Figure 4).

\section{Discussion}

Atypical gait pattern of a lack of dorsiflexion preventing heal strike and subtalar instability has been suggested as a two indicators of toe walking. Standing with full plantar surface contact on the floor was the chief indicator of the resolution of toe walking gait and fixed plantar-flexed stance in this case. High top gymshoes previously had minimized the extreme nature of the toe walking pattern but did not correct subtalar, or pronation, nor effectively lengthened Achilles through daily activities. Toe walking pattern always resumed in stance and gait without shoes. The immediate positive results in this case suggest that muscle toxicity (from either the underlying biology of ASD or the inability to voluntarily contract muscles related to dyspraxia) may have contributed to chronic fibrotic muscle stiffness from a very young age, creating a situation of obligatory toe walking and rigid plantar-flexed ankles.

The dynamics of lymphatic flow dynamics has been shown numerous times to be enhanced by the process of exercising which in turn cleans muscle and interstitium tissues [50-52]. However, this boy had severe dyspraxia that prevented his active participation in mastering typical repertoires of gross motor skills through daily experiences. His level of activity, and perhaps the degree of motor planning difficulties interfered with normal weight bearing and gross motor competencies to correct toe walking, which in turn may have assisted normal physiological pumping of lymphatic and interstitial fluids.

Chronic muscle spasms has been suggested as a etiology of toe walking gait, regardless of diagnostic group and even in the neuro-typical population. Manual stretching of a muscle may assist the lymphatic and interstitial fluid movement since the lymph vessels in the muscles can't contract spontaneously $[53,54]$. But to date these principles have yet to be applied when treating the traits of toe walking. Fibrousity and lack of fluid detoxification of muscle spasms have no mention in the literature of toe walking in ASD.

During the time treating the toe walking patterns in his therapy program, massage methods were used multiple times (with a focus on generalized massaging of muscle tissue). No positive effective upon the ankle range of motion (passive or active) occurred from any other manual therapy. There clearly was something 


\section{One Year Follow Up}

Weekly therapy sessions continued with this boy to address other areas of function and occupational performance. Language comprehension and expression skills emerged and he started to demonstrate competencies in understanding and following directions. As a result, treatment for toe-walking and fixed plantar flexion positioning was able to be advanced beyond passive range of motion into active-assisted, active range of motion activities and progressive resistive training. Heel strike continues to be absent, but active pronation-supination with hand-held assistance can be achieved, as well as AAROM of dorsi- and plantar flexion exercises.

Orthotic inserts in regular shoes now support subtalar neutral during gait.
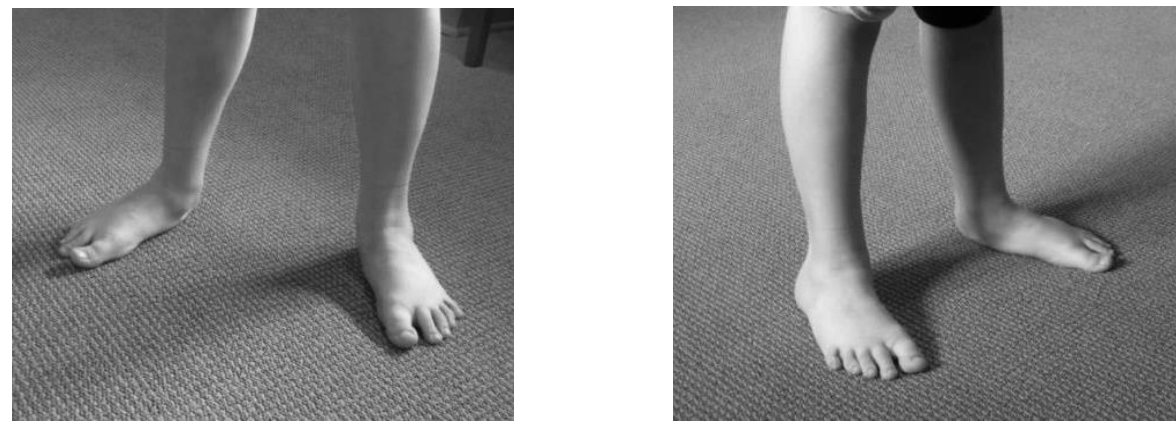

\section{Stance barefoot}

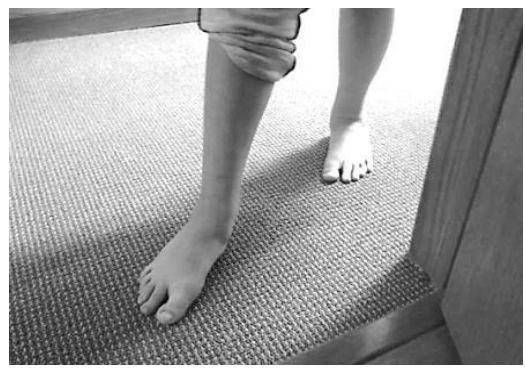

Tandem stance - reflects improved balance
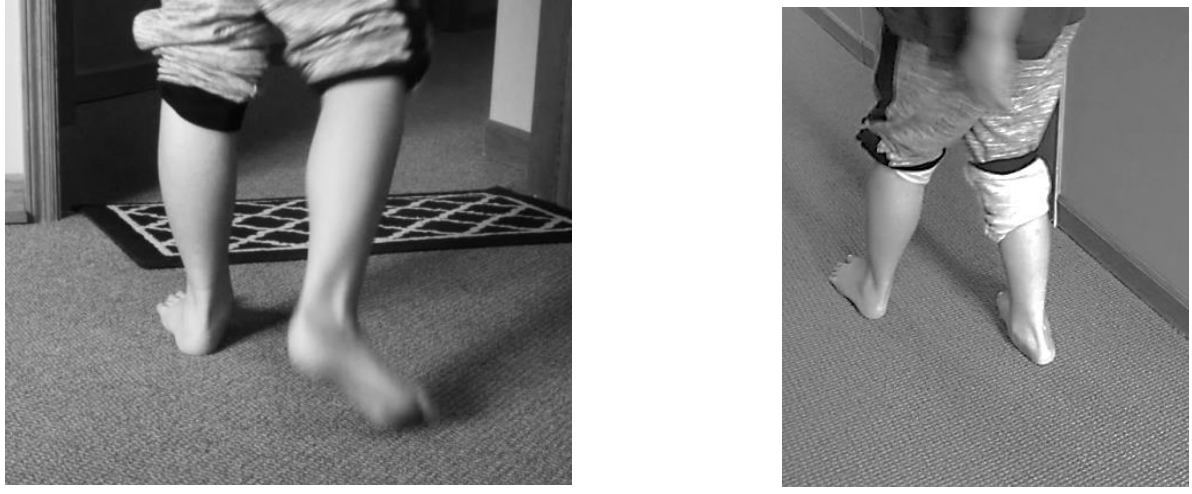

\section{Gait}

Figure 4: One year follow up: Static weight bearing stance and new gait pattern.

different about lymphatic drainage method with focus on the anatomical specificity of lymph nodes and vessel pathways that yielded these results. Manual stretching in the tradition sense does not address precise lymphatic structures or specificity of detox pathways. It is imperative to follow the lymph pathway directions accurately and apply only the slightest amount of pressure or one risks rupture to engorged nodes or injury to the system.
The impact of cognitive and language status cannot be understated as contributing factors. Prior to achieving neutral dorsiflexion from lymphatic drainage, only $0-10 \%$ cooperation and participation from him in foot [re] habilitation efforts was achieved. The patient did not have the ability to express his wants, needs, or feelings. It's possible he was experiencing pain with such tight muscles. It's also possible he either habituated to any pain or his perception of it was different than a typical 
child. Had he been able to express any pain associated with such tight gastrocnemii and soleus, his therapy course possibly would have taken a different route.

\section{Implications for Practice}

Pediatric practitioners experienced in treating persistent toe-walking gait are familiar with the complexity and confounding struggles in working with the long term consequence of, and postural challenges and the risks of contractures and deformity. Treatment planning often addresses the multitude of orthopedic and neurological aspects. This case presented with the conundrum of issues associated with primitive reflex persistence, hypotonia, lack of adaptive responses to various sensory inputs and typical gross motor play activities to would achieve foot contact for weight bearing, lack of language comprehension to fully participate with engagement in treatment for ankles, and a lack of social engagement and learning difficulties. The surprising and immediate effect of lymphatic drainage on reversing persistent and problematic toe-walking gait and fixed bilateral plantar-flexion in the patient had a lasting positive effect. Not only was neutral dorsiflexion achieved, but so many other therapy objectives could then be addressed because of rapid improvement in standing posture and gait pattern. This fact alone reduced the burden of therapeutic intervention to address the cascading effects of a lack of achieving neutral dorsiflexion for this patient.

This discovery expands on current philosophies of manual therapies by widening the scope of intervention to the 'fluids' of the human body. The mobilization and exchange of lymphatic and interstitial fluids as a detoxification of muscles was the primary action. A fluid model of human anatomy offers a tangible perspective for novel and effective treatment techniques for normalizing muscle spasms, stiffness, and inflamed tissue $[48,55]$. Various methods of manual lymphatic drainage exists [49,56-58]. The past twenty years has brought a clearer understanding of the structure and functions of the human lymphatic (and interstitial systems and their role in health and wellness [59]. More precise than older versions of massage to lymphatic fluids, the Chikly method defines anatomical pathways and mapping, specific rhythms, and depths and quality of lymph flow throughout the body [60]. The use of manual lymphatic drainage by qualified professionals is a treatment option for lymphedema, sports injuries, and fibromyalgia $[45,49,56,61-63]$.

Healthcare practitioners can readily take classes to learn to palpate the subtlety and complexity of the cellular disposal system and lymphatic anatomy, comprehensively researched and identified by Chikly [64]. Training starts with a basic evacuation technique of superficial lymphatics, and then more advanced classes explore how to work with mucosa, muscles, fas- cia, tendons, periosteum, most viscera, and nervous system structures. The technique that the therapist's hand uses in lymphatic drainage is vastly different from other traditionally recognized manual interventions. Hand pressure used in this method is extremely light, between 0.5 to 2 ounces of pressure $(28 \mathrm{~g}$ per $\mathrm{cm}^{2}$ ), only enough to stimulate the movement of fluid and enhance flow. This is believed to activate the contractions of little muscular units along lymphatic vessels, called lymphangions which assist fluid exchange without increasing blood filtration or lymph node collapse from too great of pressure [48]. There exists extensive innervation from the autonomic nervous system of these contractile units of the lymphatic system [56].

Interstitial anatomy has recently gained clarity in the understanding of its substance and structure. The interstitium is an 'organ of fluids' and is the largest organ in the body [65]. It constitutes $15 \%$ of total body mass, and $20 \%$ of total body fluid volume (three gallons of fluid). The fluid of the interstitium is believed to give rise to lymphatic fluids [66]. The interplay between the confluences of interstitial fluids with lymphatics may be difficult to discern. The manual techniques used in this case may be mobilizing both lymphatic and interstitial fluid simultaneously. The benefits of clinical reasoning and access to different population groups allowed this opportunity of discovery.

\section{Parents' perspective of treatment}

Achieving normal standing and walking for their son had been an increasing area of concern for them and they were planning on consulting a surgeon about both serial casting and surgical options. Both parents were very pleased and impressed with the immediate results of this treatment. They voiced relief over the fact that the stress of the orthopedic issues has been greatly reduced and they can focus attention and resources on his other long term needs.

\section{Patient's perspective of treatment}

The child's demeanor has changed since this single event. He has become more aware and engaged, receptive and participative in events happening around him. He now actively participates in and follows directives $75-90 \%$ of the time in his foot [re] habilitation efforts.

\section{Conclusion}

The outcomes of Chikly lymphatic drainage massage produced immediate and measureable outcomes in reducing chronic toe walking and rigid plantar-flexion stance in a young boy with ASD following one treatment. Detoxing muscles via manual evacuation of fluids through deep lymph nodes yielded positive and lasting effect. The treatment softened, relaxed, lengthened muscle tissues, and mobilized fluids of both lower extremities. The results have lasted as ev- 
idenced by photography of stance and gait one year later (Figure 4).

The strength of this case is the introduction of a new concept of working with the fluid structures of the human anatomy in approaching the quandary of persistent toe-walking gait and stance. Previous clinical trials of traditional myofascial mobilization were trialed for well over two years such as muscle stretching, reflex inhibition, forced weight-bearing, ankle positioning in shoes and other interventions yielded no or few lasting results. Obligatory toe walking had contributed to the onset of tarsal and subtalar malalignment contractures and severe Achilles contractions, but with the relaxation and softening of the gastronemii/soleus complex, these contractures have been rapidly reversed. Prognosis for normalization of gait and stance is good as active-assisted ankle strengthening has been achieved. Gaining heel strike remains to be gained, but the muscle spasms and fibrousity have not returned.

The limitations of this case are that generalizations cannot be applied to other cases of toe walking. However, the ease in which to apply manual lymphatic drainage and the gentleness of the techniques, poses little to no risk to most patients with this condition. Manual lymphatic drainage is a noninvasive method that offers qualified therapists a natural complement to existing treatment protocols.

Contraindications for lymphatic drainage was been cited and include: Acute infection or inflammatory disease process, thrombosis or phlebitis, acute heart problems as lymphatic drainage increases cardiac load, acute hemorrhage, and active malignant ailments $[48,49,56]$. For musculoskeletal and soft tissue problems this case presented, its clinical use holds value for future study.

\section{Funding}

Any conflicts of interests are implied by the author being an active clinician where this technique is but one of the therapeutic strategies utilized in the clinic setting. No competing financial interests exist. This case is not associated with any commercial entities that provide training workshops reviewed in this case.

\section{Acknowledgements}

Informed consent from parents has been given for the presentation of this case and is filed in the medical record.

The author wishes to thank all the clients who trust the wisdom of structural medicine as we continue to merge theory into day-to-day clinical practices. We are forever indebted to your tenacity and steadfastness to find and secure appropriate help.

\section{References}

1. Oetgen ME, Peden S (2012) Idiopathic toe walking. J Am Acad Orthop Surg 20: 292-300.
2. Alvarez C, De Vera M, Beauchamp R, Ward V, Black A (2007) Classification of idiopathic toe walking based on gait analysis: Development and application of the ITW severity classification. Gait Posture 26: 428-435.

3. Houck JR, Tome JM, Nawoczenski DA (2008) Subtalar neutral position as an offset for a kinematic model of the foot during walking. Gait Posture 28: 29-37.

4. Ruzbarsky JJ, Scher D, Dodwell E (2016) Toe walking: Causes, epidemiology, assessment, and treatment. Curr Opin Pediatr 28: 40-46.

5. Engström P, Tedroff K (2012) The prevalence and course of idiopathic toe-walking in 5-year-old children. Pediatrics 130: 279-284.

6. Engström P, Van'tHooft I, Tedroff K (2012) Neuropsychiatric symptoms and problems among children with idiopathic toe-walking. J Pediatr Orthop 32: 848-852.

7. Farmer SE (2003) Key factors in the development of lower limb co-ordination: Implications for the acquisition of walking in children with cerebral palsy. Disabil Rehabil 25: 807-816.

8. Eastwood DM, Dennett X, Shield LK, Dickens DR (1997) Muscle abnormalities in idiopathic toe-walkers. J Pediatr Orthop B 6: 215-218.

9. Eastwood DM, Menelaus MB, Dickens DR, Broughton NS, Cole WG (2000) Idiopathic toe-walking: Does treatment alter the natural history? J Pediatr Orthop B 9: 47-49.

10. Pomarino D, Ramírez Llamas J, Martin S, Pomarino A (2017) Literature review of idiopathic toe walking: Etiology, prevalence, classification, and treatment. Foot Ankle Spec 10: 337-342.

11. Accardo PJ, Barrow W (2015) Toe walking in autism: Further observations. J Child Neurol 30: 606-609.

12. Jeste SS (2011) The neurology of autism spectrum disorders. Curr Opin Neurol 24: 132-139.

13. Babb A, Carlson WO (2008) Idiopathic toe-walking. S D Med 61: 53-55.

14. Ming X, Brimacombe M, Wagner GC (2007) Prevalence of motor impairment in autism spectrum disorders. Brain Dev 29: $565-570$.

15. Rinehart NJ, Tonge BJ, Bradshaw JL, lansek R, Enticott PG, et al. (2006) Gait function in high-functioning autism and Asperger's disorder: Evidence for basal-ganglia and cerebellar involvement? Eur Child Adolesc Psychiatry 15: 256-264.

16. Mandell DS, Novak MM, Zubritsky CD (2005) Factors associated with age of diagnosis among children with autism spectrum disorders. Pediatrics 116: 1480-1486.

17. Vilensky JA, Damasio AR, Maurer RG (1981) Gait disturbances in patients with autistic behavior: A preliminary study. Arch Neurol 38: 646-649.

18. Kerrigan DC, Riley PO, Rogan S, Burke DT (2000) Compensatory advantages of toe walking. Arch Phys Med Rehabil 81: 38-44.

19. Williams CM, Tinley P, Curtin M (2010) Idiopathic toe walking and sensory processing dysfunction. J Foot Ankle Res 3: 16.

20. Williams CM, Tinley P, Curtin M, Nielsen S (2012) Vibration perception thresholds in children with idiopathic toe walking gait. J Child Neurol 27: 1017-1021.

21. Fanchiang HD, Geil MD, Wu J, Ajisafe T, Chen YP (2016) The effects of walking surface on the gait pattern of children with idiopathic toe walking. J Child Neurol 31: 858-863.

22. Zafeiriou DI (2004) Primitive reflexes and postural reactions in the neurodevelopmental examination. Pediatr Neurol 31: 1-8. 
23. Kindregan D, Gallagher L, Gormley J (2015) Gait deviations in children with autism spectrum disorders: A review. Autism Res Treat 2015: 741480.

24. Shetreat-Klein M, Shinnar S, Rapin I (2014) Abnormalities of joint mobility and gait in children with autism spectrum disorders. Brain Dev 36: 91-96.

25. Calhoun M, Longworth M, Chester VL (2011) Gait patterns in children with autism. Clinical Biomechanics 26: 200-206.

26. Barrow WJ, Jaworski M, Accardo PJ (2011) Persistent toe walking in autism. J Child Neurol 26: 619-621.

27. Hallett M, Lebiedowska MK, Thomas SL, Stanhope SJ, Denckla MB, et al. (1993) Locomotion of autistic adults. Arch Neurol 50: 1304-1308.

28. Stott NS, Walt SE, Lobb GA, Reynolds N, Nicol RO (2004) Treatment for idiopathic toe-walking: Results at skeletal maturity. J Pediatr Orthop 24: 63-69.

29. Herrin K, Geil M (2016) A comparison of orthoses in the treatment of idiopathic toe walking: A randomized controlled trial. Prosthet Orthot Int 40: 262-269.

30. Van Kuijk AA, Kosters R, Vugts M, Geurts AC (2014) Treatment for idiopathic toe walking: A systematic review of the literature. J Rehabil Med 46: 945-957.

31. Engelbert R, Gorter JW, Uiterwaal C, van de Putte E, Helders $P$ (2011) Idiopathic toe-walking in children, adolescents and young adults: A matter of local or generalised stiffness? BMC Musculoskelet Disord 12: 61.

32. Fox A, Deakin S, Pettigrew G, Paton R (2006) Serial casting in the treatment of idiopathic toe-walkers and review of the literature. Acta Orthop Belg 72: 722-730.

33. Brouwer B, Davidson LK, Olney SJ (2000) Serial casting in idiopathic toe-walkers and children with spastic cerebral palsy. J Pediatr Orthop 20: 221-225.

34. Davies K, Black A, Hunt M, Holsti L (2018) Long-term gait outcomes following conservative management of idiopathic toe walking. Gait Posture 62: 214-219.

35. Barkocy M, Dexter J, Petranovich C (2017) Kinematic gait changes following serial casting and bracing to treat toe walking in a child with autism. Pediatr Phys Ther 29: 270274.

36. McMulkin ML, Gordon AB, Tompkins BJ, Caskey PM, Baird GO (2016) Long term gait outcomes of surgically treated idiopathic toe walkers. Gait Posture 44: 216-220.

37. Van Bemmel AF, Van de Graaf VA, Van den Bekerom MP, Vergroesen DA (2014) Outcome after conservative and operative treatment of children with idiopathic toe walking: A systematic review of literature. Musculoskelet Surg 98: 8793.

38. Hemo Y, Macdessi SJ, Pierce RA, Aiona MD, Sussman MD (2006) Outcome of patients after Achilles tendon lengthening for treatment of idiopathic toe walking. J Pediatr Orthop 26: $336-340$.

39. Hirsch G, Wagner B (2004) The natural history of idiopathic toe-walking: A long-term follow-up of fourteen conservatively treated children. Acta Paediatr 93: 196-199.

40. Persicke A, Jackson M, Adams AN (2014) Brief report: An evaluation of TAGteach components to decrease toe-walking in a 4-year-old child with autism. J Autism Dev Disord 44: $965-968$

41. Marcus A, Sinnott B, Bradley S, Grey I (2010) Treatment of idiopathic toe-walking in children with autism using GaitSpot auditory speakers and simplified habit reversal. Research in
Autism Spectrum Disorders 4: 260-267

42. Kim A (2016) Kinesiology Taping for Rehab and Injury Prevention: An Easy, At-Home Guide for Overcoming Common Strains, Pains and Conditions. Ulysses Press.

43. Öhman AM (2013) Kinesiology taping a therapeutic tool in the paediatric population. J Nov Physiother 3: 2.

44. McGillicuddy M (2011) Massage for sport performance. Human Kinetics.

45. Vairo GL, Miller SJ, Rier NCI, Uckley WI (2009) Systematic review of efficacy for manual lymphatic drainage techniques in sports medicine and rehabilitation: An evidence-based practice approach. Journal of Manual \& Manipulative Therapy 17: 80E-89E.

46. Pacey V, de Bakker PB, Caserta AJ, Gray K, Williams CM, et al. (2016) Interventions for idiopathic toe walking. Cochrane Database Syst Rev 2016.

47. Nielsen $G$ (2016) Physical treatment of functional neurologic disorders. Handb Clin Neurol 139: 555-569.

48. Chikly B, Roberts P, Quaghebeur J (2016) Primo vascular system: A Unique biological system shifting a medical paradigm. J Am Osteopath Assoc 116: 12-21.

49. Chikly BJ (2005) Manual techniques addressing the lymphatic system: Origins and development. J Am Osteopath Assoc 105: 457-464.

50. Von der Weid PY, Zawieja DC (2004) Lymphatic smooth muscle: The motor unit of lymph drainage. Int $\mathrm{J}$ Biochem Cell Biol 36: 1147-1153.

51. Havas E, Parviainen T, Vuorela J, Toivanen J, Nikula T, et al. (1997) Lymph flow dynamics in exercising human skeletal muscle as detected by scintography. J Physiol 504: 233239.

52. Olszewski, Engeset (1985) Studies on the lymphatic circulation of humans. In: MG Johnston, Experimental Biology of the Lymphatic Circulation. Elsevier, Oxford, 395-422.

53. Mazzoni MC, Skalak TC, Schmid-Schonbein GW (1990) Effects of skeletal muscle fiber deformation on lymphatic volumes. Am J Physiol 259: H1860-H1868.

54. Skalak TC, Schmid-Schönbein GW, Zweifach BW (1984) New morphological evidence for a mechanism of lymph formation in skeletal muscle. Microvasc Res 28: 95-112.

55. Olszewski WL, Engeset A (1980) Intrinsic contractility of prenodal lymph vessels and lymph flow in human leg. Am J Physiol 239: H775-H783.

56. Chikly B (2017) Silent Waves: Theory and Practice of Lymph Drainage Therapy: With Applications for Lymphedema, Chronic Pain, and Inflammation. ( $3^{\text {rd }}$ edn $)$.

57. Millard DO (1964) Applied anatomy of the lymphatics. Health Research Books.

58. Vodder E (1936) Lymphatic drainage, a new therapeutic method Health for all, Paris.

59. Hansen KC, D'alessandro A, Clement CC, Santambrogio $L$ (2015) Lymph formation, composition and circulation: A proteomics perspective. Int Immunol 27: 219-227.

60. Chikly B, Quaghebeur J, Witryol W (2014) A controlled comparison between manual lymphatic mapping $(\mathrm{mlm})$ of plantar lymph flow and standard physiologic maps using lymph drainage therapy (Idt)/osteopathic lymphatic technique (olt). J Yoga Phys Ther 4: 173.

61. Moattari M, Jaafari B, Talei A, Piroozi S, Tahmasebi S, et al. (2012) The effect of combined decongestive therapy and 
pneumatic compression pump on lymphedema indicators in patients with breast cancer related lymphedema. Iran Red Crescent Med J 14: 210-217.

62. Asplund R (2003) Manual lymph drainage therapy using light massage for fibromyalgia sufferers: A pilot study. Journal of Orthopaedic Nursing 7: 192-196.

63. Földi E, Földi M, Weissleder H (1985) Conservative treatment of lymphoedema of the limbs. Angiology 36: 171-180.
64. (2019) Chikly Health Institute.

65. PC Benias, RG Wells, B Sackey-Aboagye, H Klavan, J Reidy, et al. (2018) Structure and Distribution of an Unrecognized Interstitium in Human Tissues. Sci Rep 8: 4947.

66. Myers TW (2013) Anatomy Trains E-Book: Myofascial Meridians for Manual and Movement Therapists. Elsevier Health Sciences. 